\title{
A FORBIDDEN SET FOR EMBEDDED EIGENVALUES
}

\author{
RAFAEL RENE DEL RIO CASTILLO
}

(Communicated by Andrew Bruckner)

\begin{abstract}
We study the problem of embedding eigenvalues to the spectrum of a Sturm-Liouville operator in the half axis when this spectrum is a perfect set. We prove the existence of an uncountable dense subset of the spectrum for which, by modifying the condition at the left or by locally perturbing the potential, it is not possible to add any eigenvalues.
\end{abstract}

\section{INTRODUCTION}

In this paper we consider Sturm-Liouville operators generated by the differential expression $l u=-u^{\prime \prime}+q(x) u$ in the half line $[0, \infty)$.

It is known that, through local perturbations of the potential or by considering arbitrary conditions at the left, it is possible to add eigenvalues anywhere in the resolvent set (see [4, Theorem 2.5.3]) or to produce an infinite number of embedded eigenvalues (see [2, Remark 5]).

Nevertheless, we prove in this paper that there exists a specific subset of the spectrum for which, assuming the spectrum of the unperturbed operator is a perfect set, it is not possible to generate embedded eigenvalues by means of the above perturbations. We also show that this "forbidden" set, which depends only on the behaviour of the potential at infinity, is a dense and uncountable set. Moreover, every point of the spectrum is a condensation point of this set.

This paper is organized as follows. In $\S 2$ we define the unperturbed operator $L$ and the perturbed operator $\widetilde{L}$. We prove that eigenvalues can appear only in points where the symmetric derivative of the spectral function of the unperturbed operator is zero. This is proved by using a theorem of Aronszajn [1]. In $\S 3$, using tools of elementary real analysis, we show that the set of points where a given series diverges is "big" in some sense. This result is crucial for proving our main theorem in the presence of only pure point spectrum. Section 4 is devoted to the proof of the main result where a theorem of Kundu [5] is applied.

Received by the editors July 31, 1992; presented to the Midwest and Southeastern Atlantic Second Joint Regional Conference on Differential Equations, November 1992, Lexington, Kentucky. 1991 Mathematics Subject Classification. Primary 34L99, 34B24, 47E05. 


\section{Preliminaries}

Consider the selfadjoint operator $L$ generated by the differential expression

$$
l u=-u^{\prime \prime}+q(x) u, \quad 0 \leq x<\infty,
$$

where $q$ is a real-valued, locally integrable function, as $L u=l u$ with domain $D(L)=\left\{u \in L_{2}(0, \infty) \mid u, u^{\prime}\right.$ are locally absolutely continuous, $l u \in L_{2}(0, \infty)$, and $\left.u(0) \cos \alpha+u^{\prime}(0) \sin \alpha=0\right\}, \quad \alpha \in[0, \pi)$.

The limit point case occurs at $\infty$, and 0 is a regular point. We shall denote the spectral function of $L$ by $\rho$. Sometimes to emphasize the dependence on $\alpha$ we shall write $L_{\alpha}$ and $\rho_{\alpha}$.

The perturbed operator $\widetilde{L}$ will be any selfadjoint realization of the differential expression

$$
\tilde{l} u=-u^{\prime \prime}+\{q(x)+v(x)\} u, \quad a \leq x<\infty,
$$

where $-\infty \leq a$ and $v(x)$ is a locally integrable function with compact support. If the limit circle case (l.c.c.) occurs at $a$, then a boundary condition will be needed.

The operator $\widetilde{L}$ is defined as $\widetilde{L} u=\tilde{l} u$ with domain $D(\widetilde{L})=\left\{u \in L_{2}(a, \infty) \mid u, u^{\prime}\right.$ are locally absolutely continuous, $\tilde{l} u \in L_{2}(a, \infty)$, and $[v, u]_{a}=0$ if we have 1.c.c. at $\left.a\right\}$.

Here $v$ is a nontrivial solution of $(\tilde{l}-\lambda) u=0(\lambda \in \mathbb{R})$ and

$$
[v, u]_{a}=\lim _{x \rightarrow a}\left(\bar{v}(x) u^{\prime}(x)-\bar{v}^{\prime}(x) u(x)\right) .
$$

See, for example, Theorem 5.8 of [6].

The following observation will be useful in the sequel.

Remark 1. If $\lambda$ is eigenvalue of $\widetilde{L}$ then, for some $\alpha \in[0, \pi), \lambda$ is an eigenvalue of $L_{\alpha}$.

Let us define

$$
D \rho(\lambda)=\lim _{\varepsilon \rightarrow 0} \frac{\rho(\lambda+\varepsilon)-\rho(\lambda-\varepsilon)}{2 \varepsilon} .
$$

Lemma 1. $\int_{-\infty}^{\infty} d \rho(\lambda) /\left(\lambda_{0}-\lambda\right)^{2}<\infty \Rightarrow D \rho\left(\lambda_{0}\right)=0$.

Proof. Assume that $\int_{-\infty}^{\infty} d \rho(\lambda) /\left(\lambda_{0}-\lambda\right)^{2}<\infty$, and, for all $\varepsilon>0$, define the interval $I_{\varepsilon}=\left[\lambda_{0}-\varepsilon, \lambda_{0}+\varepsilon\right]$. We shall denote the length of $I_{\varepsilon}$ by $\left|I_{\varepsilon}\right|$. Then we have

$$
\int_{-\infty}^{\infty} \frac{d \rho(\lambda)}{\left(\lambda_{0}-\lambda\right)^{2}} \geq \int_{I_{\varepsilon}} \frac{d \rho(\lambda)}{\left(\lambda_{0}-\lambda\right)^{2}} \geq \frac{1}{\left|I_{\varepsilon}\right|^{2}} \int_{I_{\varepsilon}} d \rho(\lambda)=\frac{1}{\left|I_{\varepsilon}\right|} \frac{\rho\left(I_{\varepsilon}\right)}{\left|I_{\varepsilon}\right|}
$$

and, therefore,

$$
\left|I_{\varepsilon}\right| \int_{-\infty}^{\infty} \frac{d \rho(\lambda)}{\left(\lambda_{0}-\lambda\right)^{2}} \geq \frac{\rho\left(\lambda_{0}+\varepsilon\right)-\rho\left(\lambda_{0}-\varepsilon\right)}{2 \varepsilon}
$$

Since

$$
\lim _{\varepsilon \rightarrow 0}\left|I_{\varepsilon}\right| \int_{-\infty}^{\infty} \frac{d \rho(\lambda)}{\left(\lambda_{0}-\lambda\right)^{2}}=0
$$

and the right-hand side of the above inequality is nonnegative, it follows that $D \rho\left(\lambda_{0}\right)$ exists and equals zero. Q.E.D. 
Lemma 2. If $\lambda_{0}$ is an eigenvalue of $\widetilde{L}$ then $D \rho\left(\lambda_{0}\right)=0$ or $\lambda_{0}$ is an eigenvalue of $L$.

Proof. Assume that $\lambda_{0}$ is an eigenvalue of $\tilde{L}$ and $D \rho\left(\lambda_{0}\right)>0$. Then from Lemma 1 it follows that $\int_{-\infty}^{\infty} d \rho(\lambda) /\left(\lambda_{0}-\lambda\right)^{2}$ is not finite. Using Theorem 4 of [1] we can conclude that $\lambda_{0}$ is not an eigenvalue of $L_{\beta}$ for $\beta \neq \alpha$. If $\lambda_{0}$ is not an eigenvalue of $L_{\alpha}$ then, from Remark 1, it follows that $\lambda_{0}$ is not an eigenvalue of $\widetilde{L}$. Q.E.D.

\section{A REAL VARIABLE RESUlt}

Let $\mathbb{N}$ denote the set of positive integers, $\mathbb{R}^{+}$the positive real numbers, and $\bar{I}$ the closure of $I$. The difference of two sets $A, B$ will be denoted by $A-B$. Recall that a set is said to be dense in itself if it is contained in the set of its limit points and that a point $p$ in a metric space $X$ is said to be a condensation point of a set $A \subset X$ if every neighborhood of $p$ contains uncountable many points of $A$.

Let $F: S \rightarrow \mathbb{R}^{+}$be an arbitrary function, where $S \subset[a, b]$ is countable and dense in itself. Define

$$
A=\left\{\xi \in[a, b]-S \mid \sum_{s \in S} \frac{F(s)}{|s-\xi|^{2}}=\infty\right\} .
$$

Lemma 3. The set $A$ defined above is uncountable, and every point of $S$ is a condensation point of $A$.

Proof. Let us consider the family of functions $\mathscr{F}:=\{\delta \mid \delta: \mathbb{N} \rightarrow\{0,1\}\}$. Choose $\delta \in \mathscr{F}$, and, for each $k \in \mathbb{N}$, denote by $\delta_{k}$ the restriction of $\delta$ to the finite set $\{1, \ldots, k\}$. We shall define for each $\delta_{k}$ an interval $I_{\delta_{k}}$.

In what follows $I_{s F}$ will denote the open interval $\left(s-F^{1 / 2}(s), s+F^{1 / 2}(s)\right)$, and the index $i$ will take the values 0,1 .

Let $I \subset[a, b]$ be an arbitrary open interval such that $S \cap I \neq \varnothing$, and choose $s_{0} \in S \cap I$. Define $I_{s_{0}}:=I \cap I_{s_{0} F}$. Select and fix two different points $a_{i}$ such that $a_{i} \neq s_{0}$ and $a_{i} \in I_{s_{0}} \cap S$. We can do this because $S$ is dense in itself.

Let $I_{a_{i}}$ be two open intervals which satisfy $a_{i} \in I_{a_{i}}, I_{a_{i}} \subset I_{s_{0}}, \bigcap_{i=0}^{1} \bar{I}_{a_{i}}=\varnothing$. Define $I_{\delta_{1}}:=I_{a_{\delta(1)}} \cap I_{a_{\delta(1)} F}$. This completes the construction for the case $k=1$. Now define $s_{1}=a_{\delta(1)}$.

Let us assume that we have defined an interval $I_{\delta_{k-1}}$ and a point $s_{k-1} \in$ $I_{\delta_{k-1}} \cap S$. Choose two points $a_{i}$ such that $a_{i} \in I_{\delta_{k-1}} \cap S$ and $a_{i} \neq s_{k-1}$, and fix two open intervals $I_{a_{i}}$ satisfying $a_{i} \in I_{a_{i}}, I_{a_{i}} \subset I_{\delta_{k-1}}, \bigcap_{i=0}^{1} \bar{I}_{a_{i}}=\varnothing$. Define $I_{\delta_{k}}:=I_{a_{\delta(k)}} \cap I_{a_{\delta(k)} F}$ and $s_{k}=a_{\delta(k)}$.

We can now define $I_{\delta_{k}}$ for each $k \in \mathbb{N}$. This definition can be done in many ways, but once we have chosen the points $a_{i}$ and the intervals $I_{a_{i}}$, the definition is unique. In order to define $I_{\delta_{k}^{\prime}}$ for any other function $\delta^{\prime}$, we choose exactly the same points $a_{i}$ and the same intervals $I_{a_{i}}$ we have chosen to construct $I_{\delta_{k}}$.

Now, for each $\delta \in \mathscr{F}$, define $B_{I}(\delta)=\bigcap_{k=1}^{\infty} \bar{I}_{\delta_{k}}$ where $\bar{I}_{\delta_{k}}$ denotes the closure of the interval $I_{\delta_{k}}$. Since $\bar{I}_{\delta_{k-1}} \supset \bar{I}_{\delta_{k}}$ for every $k \in \mathbb{N}$, it follows that $B_{I}(\delta) \neq \varnothing$.

Now define $B_{I}=\bigcup_{\delta \in \mathscr{F}} B_{I}(\delta)$ and $B=\bigcup B_{I}$ where the second union is taken over all open intervals contained in $[a, b]$ such that $I \cap S \neq \varnothing$. 
We shall prove the following:

(a) $B-S \subset A$.

(b) $B-S$ is uncountable and every point of $S$ is a condensation point of $B-S$.

Choose $\xi \in B-S$. Then $\xi \in B_{I}(\delta)=\bigcap_{k=1}^{\infty} \bar{I}_{\delta_{k}}$ for some $\delta$ and $I$. Hence, there is a sequence $s_{k} \in I_{\delta_{k}} \cap S$ such that, for all $k \in \mathbb{N},\left|\xi-s_{k}\right| \leq F^{1 / 2}\left(s_{k}\right)$. Therefore,

$$
1 \leq \frac{F\left(s_{k}\right)}{\left|\xi-s_{k}\right|^{2}} \quad \text { and } \quad \sum_{s \in S} \frac{F(s)}{|s-\xi|^{2}} \geq \sum_{k=1}^{\infty} \frac{F\left(s_{k}\right)}{\left|s_{k}-\xi\right|^{2}}=\infty
$$

implying that $\xi \in A$. This shows that (a) holds. i.e.,

To prove (b), choose an open interval $I \subset[a, b]$ and construct $B_{I}$ as before,

$$
B_{I}=\bigcup_{\delta \in \mathscr{F}} B_{I}(\delta)
$$

Since $B_{I}(\delta) \neq \varnothing$, select $x_{\delta} \in B_{I}(\delta)$ and define the function

$$
\begin{aligned}
J: \mathscr{F} & \rightarrow B_{I} \\
\delta & \rightarrow x_{\delta} \in B_{I}(\delta) .
\end{aligned}
$$

We shall see that $J$ is injective. If $\delta, \delta^{\prime} \in \mathscr{F}$ and $\delta \neq \delta^{\prime}$, then there is $k \in \mathbb{N}$ such that $\delta(k) \neq \delta^{\prime}(k)$. From the construction above it follows that $\bar{I}_{a_{\delta(k)}} \cap \bar{I}_{a_{\delta^{\prime}(k)}}=\varnothing$ and therefore $\bar{I}_{\delta_{k}} \cap \bar{I}_{\delta_{k}^{\prime}}=\varnothing$. Hence, $B_{I}(\delta) \cap B_{I}\left(\delta^{\prime}\right)=\varnothing$ and so $x_{\delta} \neq x_{\delta^{\prime}}$.

Since the set $\mathscr{F}$ is uncountable, so are $B_{I}$ and $B-S$. Since $B_{I} \subset I$, it follows that each point of $S$ is a condensation point of $A$. Thus (b) holds, and the proof of the lemma is complete. Q.E.D.

\section{The MAIN RESUlt}

In [5] Kundu proved that if:

(i) $\liminf _{x \rightarrow \xi-0} f(x) \geq f(\xi) \geq \liminf _{x \rightarrow \xi+0} f(x)$ for all $\xi \in[a, b]$,

(ii) $D f(x) \leq 0$ almost everywhere in $(a, b)$,

(iii) $\bar{D} f(x)<\infty$ except of a countable set in $(a, b)$, then $f$ is decreasing in $[a, b]$.

Here we used the notation

$$
\begin{aligned}
& \bar{D} f(x)=\limsup _{h \rightarrow 0} \frac{f(x+h)-f(x-h)}{2 h}, \\
& \underline{D} f(x)=\liminf _{h \rightarrow 0} \frac{f(x+h)-f(x-h)}{2 h} .
\end{aligned}
$$

The function $f$ is said to be decreasing if $x<y \Rightarrow f(x) \geq f(y)$. Analogously, $f$ is increasing if $x<y \Rightarrow f(x) \leq f(y)$.

We shall use this result to prove the following theorem. The spectrum of $L$ will be denoted by $\sigma(L)$.

Theorem. If for an interval $J$ the set $C=J \cap \sigma(L)$ is a perfect set, then there exists an uncountable set $B \subset C$ such that every point of $C$ is a condensation point of $B$ and, moreover, $\widetilde{L}$ cannot have eigenvalues in $B$. 
Proof. The spectral function of $L$ can be written as $\rho=\rho_{c}+\rho_{d}$ where $\rho_{c}$ is an increasing continuous function and $\rho_{d}$ is an increasing saltus function. Consider an arbitrary open interval such that $I \cap C \neq \varnothing$.

If $\rho_{c}$ is not constant in $I$ then $\rho_{c}$ is not decreasing and, applying the theorem of Kundu mentioned above, it follows that there is an uncountable set $B \subset I$ such that, if $x \in B$, then it is not possible to have $D \rho_{c}(x)=0$. Since $D \rho(x)=0$ implies $D \rho_{c}(x)=0$, it follows that the relation $D \rho(x)=0$ is not possible. In view of Lemma 2 , the two last conclusions of the theorem follow in this case. It remains to show that $B \subset C$, but this is a simple consequence of the fact that for $x$ in the resolvent set of $L$ we have $D \rho(x)=0$.

Now assume that $\rho_{c}$ is constant in $I$. In this case the spectrum is pure point in $I$ and, for any $\xi \in I$, we have

$$
\int_{-\infty}^{\infty} \frac{d \rho(\lambda)}{(\xi-\lambda)^{2}} \geq \int_{I} \frac{d \rho(\lambda)}{(\xi-\lambda)^{2}}=\int_{I} \frac{d \rho_{d}}{(\xi-\lambda)^{2}}
$$

Since $\rho_{d}$ is a saltus function, the measure generated by this function is supported on a countable set $S \subset I$.

Let us denote the measure of a point $s \in S$ by $F(s)$. Then we have

$$
\int_{I} \frac{d \rho_{d}(\lambda)}{(\xi-\lambda)^{2}}=\sum_{s \in S} \frac{F(s)}{(\xi-s)^{2}} .
$$

Since $\bar{I} \cap C=\bar{S}$ and $C$ is perfect, $S$ is dense in itself and we can apply Lemma 3, showing that for every $\xi$ in an uncountable set $A \subset I$ it happens that

$$
\int_{-\infty}^{\infty} \frac{d \rho(\lambda)}{(\xi-\lambda)^{2}}=\infty
$$

An application of Theorem 4 of [1] and Remark 1 imply now the two last conclusions of theorem. From

$$
\sum_{s \in S} F(s)=\int_{I} d \rho(s)<\infty \text { and } \sum_{s \in S} \frac{F(s)}{(\xi-s)^{2}}=\infty
$$

for $\xi \in A$, it follows that $\inf _{s \in S}|\xi-s|=0$ and therefore $A \subset C$. This can also be proven using Theorem 2.5.3 of [4]. Therefore, the remaining assertion is proven. Q.E.D.

Remark 2. The theorem holds for every perturbation $\widetilde{L}$ which has the property mentioned in Remark 1.

Remark 3. Using Theorem 1 of Donoghue [3] instead of Theorem 4 of Aronszajn [1], a similar result can be obtained for perturbations $H_{\alpha}=H_{0}+\alpha P$ of a selfadjoint operator $H_{0}$, where $P$ is a selfadjoint projection on a fixed normalized element and $\alpha \in \mathbb{R}$.

Remark 4. If $\lambda \in B$ then $l u=\lambda u$ has no solution which lies in $L_{2}$ near $\infty$.

\section{ACKNOWLEDGMENT}

I thank Professor Don Hinton for a stimulating conversation and the referee for useful comments. 


\section{REFERENCES}

1. N. Aronszajn, On a problem of Weyl in the theory of singular Sturm-Liouville equations, Amer. J. Math. 79 (1957), 597-610.

2. R. R. del Rio Castillo, Embedded eigenvalues of Sturm-Liouville operators, Comm. Math. Phys. 142 (1991), 421-431.

3. W. F. Donoghue, On the perturbation of spectra, Comm. Pure Appl. Math. 18 (1965), 559-579.

4. M. S. P. Eastham and H. Kalf, Schrödinger type operators with continuous spectra, Pitman Research Notes in Math., vol. 65, Longman Sci. Tech., Harlow, 1982.

5. N. K. Kundu, On some properties of symmetric derivatives, Ann. Polon. Math. 30 (1974), 9-18.

6. J. Weidmann, Spectral theory of ordinary differential operators, Lecture Notes in Math., vol. 1258, Springer-Verlag, New York, 1987.

Departamento de Métodos Matemáticos y Numéricos, IIMAS-UNAM, Apartado Postal 20-726, MÉxico, D. F., 01000, MÉxico

E-mail address: delrio@redvax1.dgsca.unam.mx 